\title{
Auger Stimulated Ion Desorption of Negative Ions via $K$-Capture Radioactive Decay
}

\author{
S. V. Verkhoturov, E. A. Schweikert,* Victor Chechik, ${ }^{\dagger}$ Rajaram C. Sabapathy, and Richard M. Crooks \\ Department of Chemistry, Texas A\&M University, College Station, Texas 77842-3012 \\ E. S. Parilis \\ Division of Physics, Mathematics and Astronomy, 200-36, California Institute of Technology, Pasadena, California 91125
}

(Received 6 December 2000; published 29 June 2001)

\begin{abstract}
We report on Auger stimulated ion desorption via Coulomb explosion from surface self-assembled alkylthiol and fluorocarbon molecular layers, triggered by $K$-capture decay of an imbedded radioactive ${ }^{55} \mathrm{Fe}$ atom. The charge state of the ejecta is determined by charge exchange in binary atomic collisions in bulk and electron tunneling outside the solid, as well as by fragmentation of electronically excited molecules or molecular fragments. We describe the first nonbeam experiments documenting positive and abundant negative ion desorption due solely to core electron excitation after radioactive decay.
\end{abstract}

DOI: $10.1103 /$ PhysRevLett.87.037601

It has long been recognized that inner shell ionization causes charging and electronic excitation within a molecular volume [1]. In free molecules, core electron excitation leads to Coulomb explosion and, hence, molecular fragmentation [2]. Later attention has shifted to the effects of the Auger cascade in chemisorbed molecules on ordered surfaces [3]. Here, core electron excitation, effected with synchrotron radiation or electron beams, causes desorption of chemisorbed species as ions or neutrals and can result in the selective breaking of bonds [4-6]. Auger transitions leading to ion emission are also caused by multiply charged ion surface interactions [7]. Most studies have been concerned with the emission of positive ions [8]. Negative ion emission attributed to the Auger process has been reported recently in electron beam experiments [9].

This study presents the first nonbeam experiments documenting positive and abundant negative ion desorption due solely to core electron excitation after radioactive decay. Each Auger cascade was observed as a temporally and spatially isolated event in a well-defined chemical environment. With this approach, we could also address the question of how the Auger cascade can cause abundant negative ion emission.

Our observations are based on core shell ionization via radioactive $K$-capture decay. We incorporated radioactive ${ }^{55} \mathrm{Fe}$ atoms at the terminus of a self-assembled monolayer or at the junction of two molecules forming a bilayer. This approach opens the possibility of studying the physical and chemical effects of a quasistationary, transient high charge state ion in selected molecular environments. "Ancillary" effects encountered with ion, electron, or photon beams, such as emission of photoelectrons, ion-atom collisions, and sputtering, which in turn cause ion emission, are avoided. Conversely, observations relying on the decay of radioisotopes must contend with a data rate controlled by the number of radioisotopes (monolayer amounts in our case) and by the ratioactive half-life.

The transition, ${ }^{55} \mathrm{Fe}+e^{-} \rightarrow{ }^{55} \mathrm{Mn}+\nu$, is a ground to ground nuclear state transition. It results in the emission of
PACS numbers: 79.90.+b, 23.40.-s, 32.80.Hd, 34.50.Gb

a neutrino and a daughter nucleus with a recoil energy of $3.2 \mathrm{eV}$, which is approximately 40 times smaller than the potential energy in the Coulomb explosion created by the Auger cascade. Thus, neutrino-recoil induced desorption, which has been observed with other radioisotopes [10], is negligible in the case considered in our report.

The experiments were run in the single electron or ion detection and event-by-event counting mode. The products of desorption were identified via time-of-flight mass spectrometry, with the start provided by the KLL or $L M M$ Auger electrons. A scheme of the experimental setup is provided in Fig. 1. The sample surfaces were biased to $\pm 3 \mathrm{kV}$. The Auger electrons used to start the measurements were selected with a combination of a magnetic field and an electrostatic grid assembly. Acid-terminated alkyl and aromatic thiol monolayers were prepared on goldcoated silicon wafers as described previously [11]. These self-assembled monolayers (SAMs) were then treated with solutions containing ${ }^{55} \mathrm{Fe}$ to form carboxylate-coordinated Fe(III)-terminated SAMs [Figs. 2 and 3(a)]. Bilayers [Fig. 3(b)] were produced by capping Fe(III)-terminated SAMs with partially fluorinated $n$-alkylthiols [12]. The ${ }^{55} \mathrm{Fe}$ (half-life, $2.7 \mathrm{yr}$ ) was obtained as a solution of $\mathrm{FeCl}_{3}$ $(13.5 \mathrm{mCi} / \mathrm{mg})$ from NEN Life Science Products, Inc.

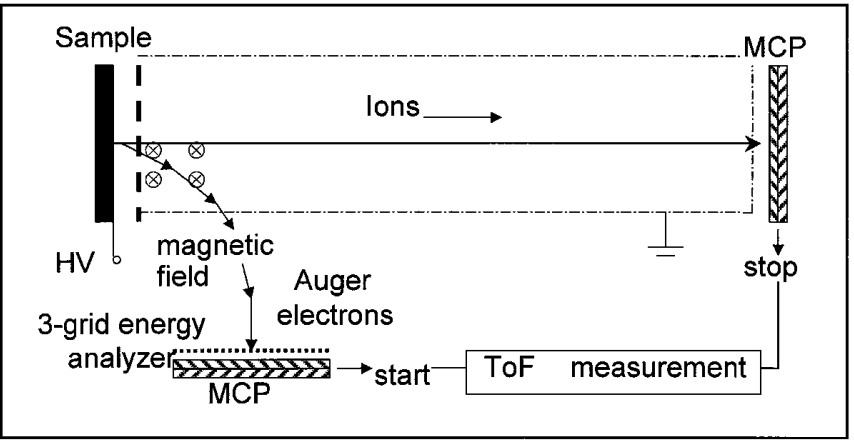

FIG. 1. Experimental setup. The sample surface was biased to $\pm 3 \mathrm{kV}$. Microchannel plates (MCP) were used both for detection of ions and electrons. 


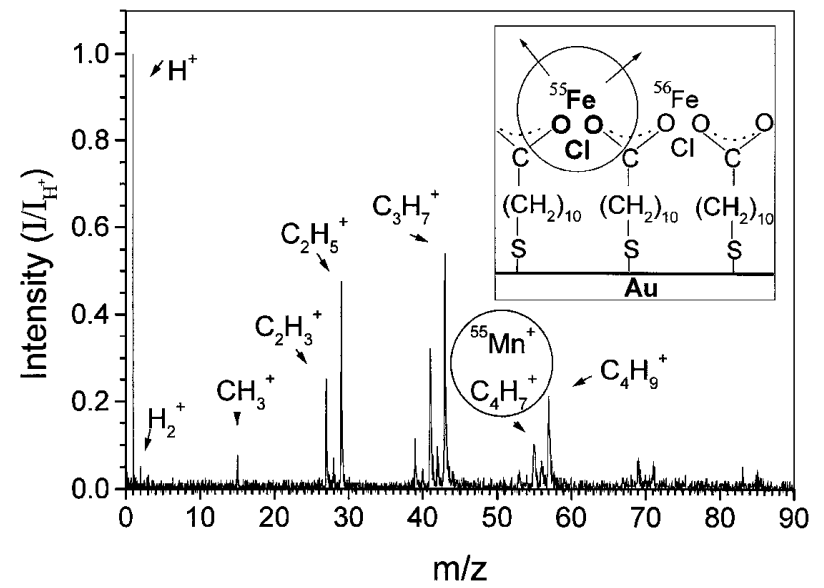

FIG. 2. Mass spectrum of positive ions emitted from a Fe(III)capped SAM, with the radioactive ${ }^{55} \mathrm{Fe}$. The sketch on the right side shows the SAM configuration.

A positive ion spectrum from a mercaptoundecanoic acid SAM coordinated to ${ }^{55} \mathrm{Fe}$ is shown in Fig. 2. The mass spectrum contains peaks of $\mathrm{H}^{+}$and $\mathrm{C}_{n} \mathrm{H}_{m}{ }^{+}$ions, but ${ }^{55} \mathrm{Mn}$ could not be identified due to a mass overlap with $\mathrm{C}_{4} \mathrm{H}_{7}{ }^{+}$. The absence of $\mathrm{O}^{+}, \mathrm{OH}^{+}$suggests that, if these ions are created, they undergo neutralization due to their high ionization potential. We invoke here surface exchange processes, which occur irrespective of the process initiating the ion emission [13]. The positive ion yield per decay of ${ }^{55} \mathrm{Fe}$ can be estimated as follows. As ${ }^{55} \mathrm{Fe}$ decays via $K$ capture with the rate $\sim 10 \mathrm{dps}$, and the ion emission rate of $\sim 10^{-3}$ ions per second, the positive ion yield is $\sim 10^{-4}$ ions/decay. This value is in agreement with the ion yields observed for metal and alkali halide atoms $K$-shell photoionization. For example, the yields of $\mathrm{Na}^{+}$ and $\mathrm{F}^{+}$have been reported to be smaller than $10^{-4}$ ions per core ionization [14].

Much more information can be obtained when observing the emission of negative ions. Figure 3(a) shows a spectrum obtained from a 4-mercaptobenzoic acid SAM coordinated to ${ }^{55} \mathrm{Fe}$. There is a very intense peak due to $\mathrm{H}^{-}$ with some indication also of emission of $\mathrm{O}^{-}, \mathrm{OH}^{-}, \mathrm{C}_{n} \mathrm{H}_{m}{ }^{-}$, and $\mathrm{Cl}^{-}$. To explore the mechanism of negative ion emission, we capped the Fe(III)-terminated SAM with partially fluorinated $n$-alkylthiol [see Fig. 3(b)]. The integrity of the bilayer was verified by reflection IR spectrometry and $\mathrm{x}$-ray photoemission spectroscopy (XPS). The negative ion emission from the bilayer is quite different from that from the monolayer. Figure 3(b) shows peaks due to $\mathrm{H}^{-}$, $\mathrm{O}^{-}, \mathrm{C}_{2} \mathrm{H}^{-}, \mathrm{Cl}^{-}, \mathrm{S}^{-}, \mathrm{F}^{-}$, and $\mathrm{F}_{2}^{-}$. The ions $\mathrm{H}^{-}, \mathrm{O}^{-}$, $\mathrm{C}_{2} \mathrm{H}^{-}, \mathrm{Cl}^{-}$, and $\mathrm{S}^{-}$are from the vicinity of ${ }^{55} \mathrm{Fe}$. The difference with a SAM spectrum [Fig. 3(a)] is that the bilayer yields $\mathrm{S}^{-}, \mathrm{F}^{-}$, and $\mathrm{F}_{2}{ }^{-}$while the emission of $\mathrm{C}_{n} \mathrm{H}_{m}{ }^{-}$ions is reduced. The negative ion yield is higher than that of the positive ions. The yield of $\mathrm{H}^{-}$is $\sim 1$ ion/decay. The yield of $\mathrm{F}^{-}$is $\sim 0.015$ ion/decay; those pertaining to other ions did not exceed $10^{-3}$ ions/decay.
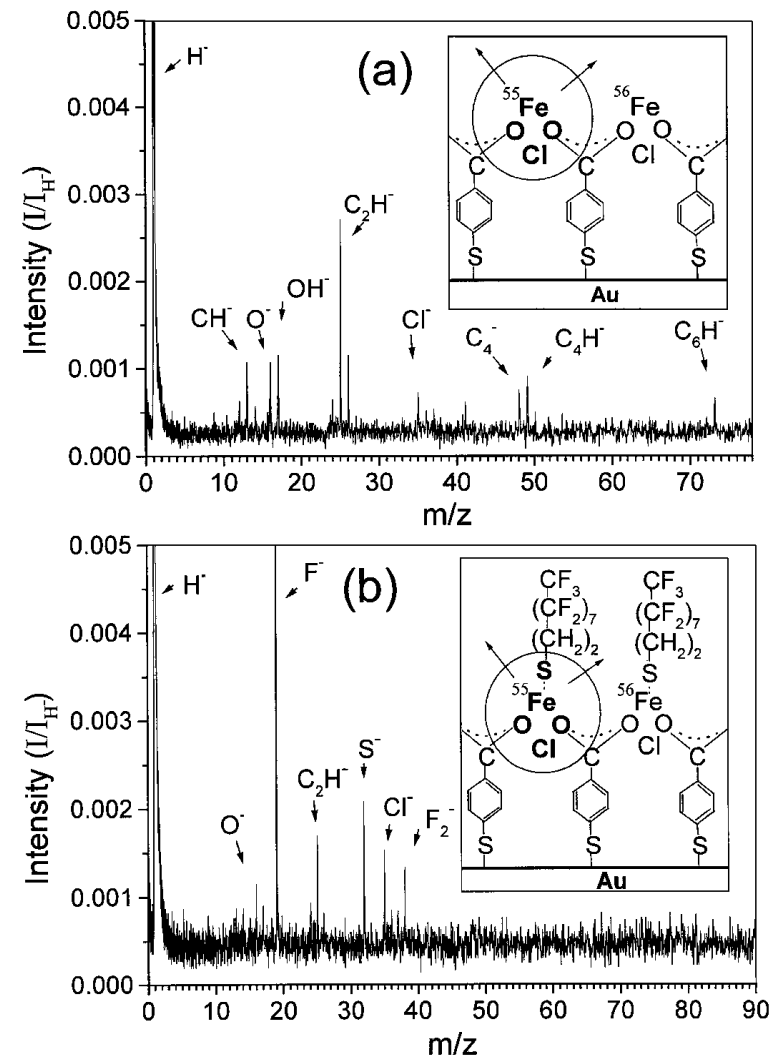

FIG. 3. (a) Mass spectrum of negative ions emitted from a $\mathrm{Fe}$ (III)-capped SAM with the radioactive ${ }^{55} \mathrm{Fe}$. (b) Mass spectrum of negative ions emitted from a bilayer. The sketches on the right side show the layer configurations.

We have verified the integrity of our observations with two control experiments. The first one involved SAMs and bilayers with nonradioactive $\mathrm{Fe}$ atoms. We could not obtain any mass spectrum in the absence of ${ }^{55} \mathrm{Fe}$; thus, possible contributions from field emission or spontaneous desorption were negligible under our experimental conditions. Another potential cause for misinterpretation is a possible contribution to desorption from nanoprecipitates containing iron, which deposit on the surface when the substrate is immersed in the $\mathrm{FeCl}_{3}$ solution. XPS suggests that these nanoparticles exist as $\mathrm{Fe}_{2} \mathrm{O}_{3}$, and atomic force microscopy (AFM) indicates they are $\sim 100 \mathrm{~nm}$ in diameter and cover $\sim 1 \%$ of the surface. The presence of the $\mathrm{Fe}_{2} \mathrm{O}_{3}$ nanoparticles results in a ${ }^{55} \mathrm{Fe}$ activity, measured with $K L L$ electrons, typically 3 times higher than that expected from a Fe monolayer. Note that only $0.001 \%$ of the total number of $\mathrm{Fe}$ atoms are radioactive (specific activity of our sample is $13.5 \mathrm{mCi} / \mathrm{mg}$ ). One could suggest that ${ }^{55} \mathrm{Fe}$ decaying in the codeposited $\mathrm{Fe}_{2} \mathrm{O}_{3}$ generates a flux of Auger and secondary electrons that might cause electron stimulated desorption. This possibility was tested by preparing SAMs consisting of $\mathrm{HS}\left(\mathrm{CH}_{2}\right)_{10} \mathrm{CH}_{3}$ and $\mathrm{HS}\left(\mathrm{C}_{6} \mathrm{H}_{5}\right)$ which do not specifically bind $\mathrm{Fe}$ (that is, there is no acid group). Under these conditions, XPS and AFM still indicate the presence of $\mathrm{Fe}_{2} \mathrm{O}_{3}$ and Auger electron count is similar to 


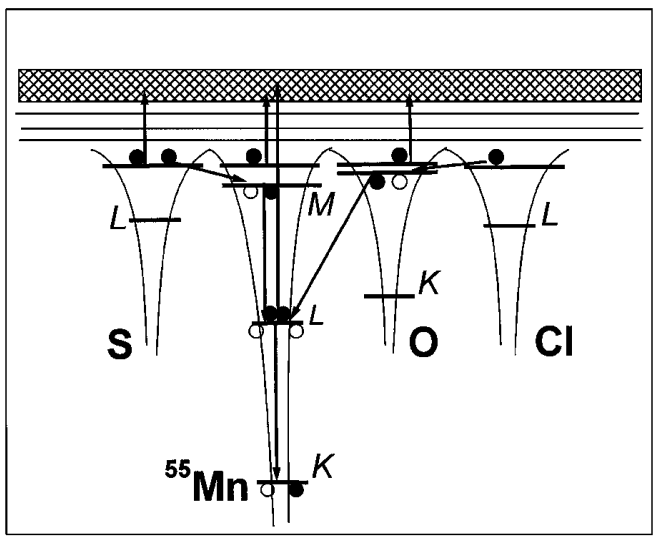

FIG. 4. Schematic diagram of Auger transitions initiated by the $K$-capture decay. The initial "vertical" intra-atomic Auger transitions are accompanied by "horizontal" interatomic Auger events involving the atoms neighboring the daughter ${ }^{55} \mathrm{Mn}$ ion.

that obtained from the thin films designed to complex Fe. However, mass spectra obtained from the $\mathrm{HS}\left(\mathrm{CH}_{2}\right)_{10} \mathrm{CH}_{3}$ and $\mathrm{HS}\left(\mathrm{C}_{6} \mathrm{H}_{5}\right)$ monolayers showed only peaks of $\mathrm{H}^{-}$at an intensity barely above the noise level. Therefore, the spectra presented in Figs. 2 and 3 are attributed solely to Auger cascades triggered by the $K$-capture decay of ${ }^{55} \mathrm{Fe}$ atoms placed specifically at the sites defined by the monolayer and bilayer templates.

Based on the experimental evidence, we offer two possible mechanisms of ion emission. The first one is Auger stimulated Coulomb explosion of a charged domain. The second mechanism is desorption and in-flight fragmentation of excited molecules emerging from the surface. Within $10^{-14} \mathrm{~s}$ after the $K$ vacancy is created in the radioactive atom, a $K$ - $L L$ Auger electron is emitted and an Auger cascade in the daughter atom ${ }^{55} \mathrm{Mn}$ is developed leading to total emission of $q$ Auger electrons, carrying away the main part of the accumulated energy $E_{K}=$ $6.54 \mathrm{KeV}$. The rest of the energy $W_{q}$ is equal to the total ionization energy of a $q$-charged ion. In an isolated atom this would end up in creation of a multiply charged ion with the positive charge $q$, up to +7 and the average charge $q=+5$. If the radioactive atom is imbedded in an organic molecule or in a solid, the initial "vertical" intra-atomic Auger transitions would be accompanied by "horizontal" interatomic Auger events involving the neighboring atoms (Fig. 4) [15]. Usually the participating electrons are the ones that provide the chemical bonds, and the Auger rate of the interatomic transitions is comparable with those for the intra-atomic ones. The energy $W_{q}$ is a source of an additional $p \approx 5$ Auger electrons emitted. There is a close analogy with the Auger neutralization of a slow multiply charged ion entering the solid from outside [16]. The final $q+p$ vacancies, the exact numbers depend on Auger cascade development, are spread among the neighboring atoms forming within the time $\tau_{a} \approx 10^{-13} \mathrm{~s}$ a domain of positive charge. The Coulomb repulsion energy of the domain $E_{c}=E_{K}-E_{a, r}-W_{q+p}$, where $E_{a, r}$ is the total energy of the Auger electrons and photons emitted, and $W_{q+p}$ is the sum of ionization energy for all $(q+p)$ ions created. $E_{c}$ is the source of radiation damage in solids and biological substances or sputtering and secondary ion emission if the radioactive atom has been imbedded close to the surface.

For the proximate neighborhood of the radioactive atom shown in Fig. 3(b) and with $q+p \approx 10$ positive charges distributed among the oxygen, carbon, and sulfur atoms in the closest vicinity of the positively charged daughter ion ${ }^{55} \mathrm{Mn}^{+}$, the accumulated Coulomb repulsion energy equals $E_{c} \approx 100 \mathrm{eV}$. Thus, $E_{c} / E_{K} \approx 2 \times 10^{-2}$, i.e., the Coulomb energy of the charged domain, takes just about $2 \%$ of the total initial energy input. The domain atoms stripped of the bonding electrons and charged positively are subject to Coulomb explosion that provides a radial expansion in all directions, including the direction outside the surface. The repulsion energy per ion $E_{r}=E_{c} /(q+p)$ is converted into hyperthermal kinetic energy that does not exceed a few eV per ion. On their way toward the surface the ions experience some chargeexchanging collisions with other atoms of the solid that occur on a mean path $\lambda_{0}=\left(\sigma_{0} n\right)^{-1}$, where $\sigma_{0}$ is the cross section of charge exchange and $n$ is the atomic density of the substance. The kinetic energy $E_{a}=\varepsilon E_{r}$ gained by the atom within the path $\lambda_{0}$, i.e., as long as it keeps the positive charge, is a fraction of $E_{r}$, the larger the longer is $\lambda_{0}$. Given the density of the self-assembled layers, $n=0.03-0.05$ atoms $/ \AA^{3}$ and $\sigma_{0} \approx 0.5 \AA^{2}$ for the hyperthermal energy [17], we calculate that the ions are neutralized within five to seven interatomic distances, that gives a value $\varepsilon \geq 3 / 4$, which means that the flux of neutral atoms and molecules emerging from the explosion region retains the main part of the Coulomb repulsion energy acquired in the "first push." Such atoms as Cl, H, O, and S [Fig. 3(b)], when neutralized, have no chance to be reionized positively at their $\mathrm{eV}$ energy. Therefore the neutral ejecta are detected only when they acquire a negative charge.

The conversion of neutral atoms into negative ions occurs in electron capture via charge exchange in binary collisions in the bulk, as well as by electron tunneling outside the surface. The electron capture occurs on a mean path $\lambda_{-}=\left(\sigma_{-} n_{a}\right)^{-1}$, where $\sigma_{-}$is the cross section of electron capture, depending on the electron affinity of the atoms and their velocity $v_{a}$, while $n_{a}$ is the atomic density of anions. As $\sigma_{-}<\sigma_{0}$, the distance $\lambda_{-}>\lambda_{0}$. The mean path of converting the positive ion into a negative one $\lambda_{ \pm}=\lambda_{0}+\lambda_{-}$is comparable to the total path $L$ of escape at the given surface layer geometry [Fig. 3(b)]. The estimate shows that $\lambda_{0}<L<\lambda_{ \pm}$. Thus, after experiencing the Coulomb explosion push, the positive ions are significantly neutralized on their way toward the surface, but the neutral flux is converted only partially into negative ions. After the neutral atoms leave the surface, they 
keep capturing electrons via tunneling with the final negative ionization degree of the flux depending on the electron affinity.

A special case is the emission of negative hydrogen ions, which have a low electron affinity. Our evidence of the $\mathrm{H}^{-}$emission with a yield close to 1 ion/decay supports the mechanism of extensive electron capture by slow ions. Our surfaces can be considered as a system of oxygen anions and iron cations for monolayers [Fig. 3(a)] and fluorine anions and carbon cations for bilayers [Fig. 3(b)]. In addition to the Coulomb explosion mechanism of hydrogen emission, which proved to be efficient [17], this unique combination of surface states gives a high yield of $\mathrm{H}^{-}$by a mechanism similar to that proposed for surface hydrogen scattering [18]. The authors of Ref. [18] observed the effective $\mathrm{H}^{+}$neutralization and $\mathrm{H}^{-}$formation at low (hyperthermal) energy backscattering on alkali halides and oxides. The electron attachment occurs in a "transient chemisorption state" on the surface, and the cationic site is very efficient for creation of $\mathrm{H}^{-}$.

A second way of molecular ion emission begins with the Auger-stimulated breaking of the bond between S and the partially fluorinated $n$-alkylthiol on the top. The bond breaking causes the emission of $\left(\mathrm{CH}_{2}\right)_{2}$ and the fluorocarbon molecule [Fig. 3(b)]. The appearance of an intense peak of $\mathrm{S}^{-}$in the mass spectrum confirms this mechanism. The molecule gets the push and the excitation from the Coulomb explosion, and then undergoes fragmentation via a process similar to the dissociative electron attachment. The difference from the usual electron attachment lies in the fact that the energy for the decay is not obtained from the electron-molecule interaction. Thus, the molecule is excited before the charge exchange process occurs. Then the negatively charged molecule undergoes fragmentation. The main pathways of fragmentation of fluorocarbon molecules via dissociative electron attachment are the emission of ions $\mathrm{F}^{-}$and $\mathrm{F}_{2}{ }^{-}$[19]. This is due to the high electron affinity of the fragments. The other fragment of this molecule is a $\mathrm{C}_{2} \mathrm{H}^{-}$. The molecule of $\mathrm{C}_{2} \mathrm{H}$ has a very high electron affinity, $3.73 \mathrm{eV}$, and the pathway of fragmentation leading to the emission of $\mathrm{C}_{2} \mathrm{H}^{-}$should be also favorable. This behavior of the top chain molecule can explain the mass spectrum in Fig. 3(b). While the atoms $\mathrm{O}$, $\mathrm{S}, \mathrm{Cl}$, and $\mathrm{H}$ get a push as neutrals or positive ions from Coulomb explosion, and then undergo efficient negative ionization, the emission of the ions $\mathrm{F}^{-}$and molecules $\mathrm{F}_{2}{ }^{-}$ and $\mathrm{C}_{2} \mathrm{H}$ is a result of top chain molecule fragmentation.

Our experiments document positive and abundant negative ion desorption due solely to core electron excitation. By imbedding ${ }^{55} \mathrm{Fe}$ in a SAM as a bilayer, we can observe the physical and chemical effects of the Auger cascade in a structured supramolecular volume. While we can offer plausible mechanisms for surface ionization-desorption including the preferential emission of negative ions, the abundant emission of $\mathrm{H}^{-}$is a surprising observation. The experiments described here can be viewed as a direct probe into the initial phase of radiation damage due to radioactive decay in an organic or biomolecular substance. This initial work suggests the feasibility of studying the effects of an isolated Coulomb explosion at specified sites in nanostructures.

This study was supported by the Robert A. Welch Foundation (A-1482). R. M. C. acknowledges support from the National Science Foundation (CHE-9818302). In addition, we thank J. Alvarez for sample preparation.

*Corresponding author.

Email address: schweikert@mail.chem.tamu.edu

${ }^{\dagger}$ Current address: Department of Chemistry, University of York, Heslington, York YO10 5DD, United Kingdom.

[1] T. A. Carlson in Desorption Induced by Electronic Transitions: DIET I, edited by N. H. Tolk et al. (Springer-Verlag, Berlin, 1983), p. 169.

[2] T. Carlson and R. M. White, J. Chem. Phys. 38, 2930 (1963).

[3] M. L. Knotek and P. J. Feibelman, Phys. Rev. Lett. 40, 964 (1978).

[4] S. P. Frigo et al., Phys. Rev. Lett. 80, 2813 (1998).

[5] R. Romberg et al., Phys. Rev. Lett. 84, 374 (2000).

[6] D. E. Ramaker, T. E. Madey, and R. L. Kurtz, Phys. Rev. B 38, 2099 (1988).

[7] T. Schenkel et al., Phys. Rev. Lett. 83, 4273 (1999).

[8] T. E. Madey, Surf. Sci. 299/300, 824 (1994).

[9] A. M. Lanzillotto, T.E. Madey, and R. A. Baragiola, Phys. Rev. Lett. 67, 232 (1991).

[10] L. Zhu et al., J. Vac. Sci. Technol. A 12, 2037 (1994).

[11] M. Wells et al., Langmuir 12, 1989 (1996).

[12] S. D. Evans et al., J. Am. Chem. Soc. 113, 5866 (1991).

[13] E. S. Parilis et al., Atomic Collisions on Solid Surfaces (North-Holland, Amsterdam, 1993), p. 207.

[14] C. C. Parks et al., Phys. Rev. B 28, 4793 (1983).

[15] E. S. Parilis, The Auger Effect (Fan, Tashkent, 1969), translated from Russian for Oak Ridge National Laboratory, P.O. 34B-88372, Release U-43.

[16] I. S. Bitensky and E. S. Parilis, J. Phys. C 50, 227 (1989).

[17] I. Bitensky, E. Parilis, S. Della-Negra, and Y. Le Beyec, Nucl. Instrum. Methods Phys. Res., Sect. B 72, 380 (1992).

[18] R. Souda et al., J. Chem. Phys. 110, 2226 (1999).

[19] P. W. Harland and J. C. J. Thynne, Int. J. Mass Spectrom. Ion Phys. 9, 253 (1972). 